\title{
Design, Simulate and Construct a Fingerprints Attendance System with Data Logging
}

\author{
A. Basila ${ }^{1 *}$, A. Danladi ${ }^{2}$ \\ ${ }^{1,2}$ Department of Pure and Applied Physics, Adamawa State University, Mubi, NIGERIA.
}

\begin{abstract}
Because of its uniqueness and accuracy over time, fingerprint has been used for identification for many years, more recently being automated due to advancement in computing capabilities, fingerprint identification is one of the most well-known and popular biometric identification systems. The methodology comprised of a power supply, input voltage to the LM7805 should be at least $2 \mathrm{v}$ greater than the required $5 v$ output according to its rating from the datasheet; hence it requires an input voltage of atleast $7 v$. Hence, 9v battery was adopted with current rating of 1 A for convenience. The LM7805 voltage regulator IC was used since we needed 5v. The Fingerprints Attendance System with Data-login was designed, simulated and implemented/constructed, and was able to address the attendance issues in Adamawa State University, Mubi by the following means: no time waste as the attendance is taken during lecture without intervention of the lecturer, managing the attendance is automated, no chance for buddy signing, real time attendance capture, evaluate level of attendance for students automatically. Also, this system can easily be applied for examination attendance and monitoring.
\end{abstract}

Keywords: Biometrics, Fingerprint, Identification.

\subsection{INTRODUCTION}

Owing to their uniqueness and accuracy over time, fingerprint identification has been used for identification for many years, most recently being automated due to advancements in computing capabilities. Fingerprint identification is one of the most well-known and popular biometric identification systems.

The fingerprint system seems to be the most cost effective and easy to use among all of the biometric system with no health side effects [1]. Biometrics, especially in information technology encompasses methods to analyze physical and behavioral Identities to extract unique features for identification or monitoring purposes. Various physical features including faces, eyes, fingers, hands, veins, ears and teeth can be used by this technology, and characteristics such as gaits or voice patterns are still being investigated and analyzed as part of the wider biometrics field. However, fingerprint biometrics is more accurate, unique, Immutable and acceptable than any other biometrics system [2].

Fingerprint being the oldest and easily available trait of biometrics, offers infallible means of personal identification. The matching accuracy using fingerprint has been shown to be very high as compared to other existing biometric traits. Unlike face and voice patterns, fingerprints are persistent with age and can't be easily distinguished. Therefore, fingerprint is one of the most

*Corresponding author (Tel: +234 (0) 7035873463)

Email addresses: basilaphysicist@gmail.com (A. Basila), deshalangs3g@yahoo.com (A. Danladi) researched and matured field of biometric authentication [3].

According to Chaudhari et al., [4], there are two different ways to resolve a person's identity: verification and identification. Verification is based on confirming or denying a person's claimed identity and answers question "Am I whom I claim I am?" In the case of identification, one has to establish a person's identity answering the question "Who am I?" A biometric system is essentially a pattern recognition system that compare unique physiological or behavioral characteristic possessed by the user with prerecorded data. As such it can be used in both approaches for identification. Identification is "oneto-many" process of determining a person's identity by performing matches against multiple biometric templates. A fingerprint is a pattern of ridges and valleys on the surface of a fingertip. The endpoints and crossing points of ridges are called minutiae. It is widely accepted assumption that the minutiae pattern of each finger is unique and does not change during person's lifetime. Ridge endings (terminations) are points where the ridge curve terminates, and bifurcations are where the ridge splits from a single path to two paths as a Y-junction [5].

\subsection{Microcontrollers}

Microcontroller is a computer on single integrated circuit that includes a CPU, RAM, some form of ROM, and I/O ports. It may be considered as a single-chip computer. Microcontrollers are usually used in devices from all aspect of life. Any device that measures, stores, 
controls, calculates or displays information is a candidate for putting microcontroller inside.

The first microcontroller was by two engineers; Gary Boone and Michael Cochran, at Texas according to the Smithsonian Institution. They created the TMS1000, which was a 4-bit microcontroller with built-in ROM and RAM. The microcontroller was used internally at TI in its calculator products from 1972 and was refined over the years. Intel later developed many important microcontrollers, two of which are the 8048 (in the year 1976), and 8051 (in the year 1980). The 8051 became one of the most popular microcontroller families, which many of its architecture are still being produced today, making the 8051 one of the most long-lived electronics designs in history [6].

During the 1990s, microcontrollers with electrically erasable and programmable ROM (EEPROM) memories, such as flash memory, became available. These microcontrollers could be programmed, erased and reprogrammed using only electrical signals. Prior to the electrically reprogrammable devices, microcontrollers often required that the device be removed from its circuit, slowing software development and making the effort more expensive. Overcoming this limitation, microcontrollers were able to be programmed and reprogrammed while in circuit, so devices with microcontrollers could be upgraded with new software without having to be returned to the manufacturer. Many current microcontrollers, such as those available from microchip and Atmel, incorporate flash memory technology.

In addition to general purpose devices, specialized microcontrollers are being produced for areas such as automotive, lighting, communications, and lowpower consumer devices. Microcontrollers have also become smaller and more powerful. Example, in 2010, Atmel announced a flash microcontroller in a package measuring $2 \mathrm{~mm}$ by $2 \mathrm{~mm}$. These tiny microcontrollers are small and cheap enough to be used in products such as toys and toothbrushes. [7]. The aim and objectives of the paper is to design, simulate and construct a fingerprints attendance system with data logging.

\subsection{MATERIALS AND METHOD}

Fingerprint identification system is mainly divided into three modules; fingerprint image preprocessing, minutiae extraction and minutiae matching [8]. The input fingerprint image is processed for skeleton image by the fingerprint image preprocessing stage and subsequently processed by minutiae extraction stage for extracting minutiae using crossing number concept. After minutiae extraction stage, if input fingerprint image is processed for enrollment then the skeleton image is saved as template fingerprint image in database, otherwise skeleton image is given to matching stage. In matching stage system compares skeleton image with template fingerprint images from database and make decision whether input fingerprint match or not.

\subsection{Power supply}

Power supply is an essential part of any electronic device. This is because if the power supply is not powerful enough to meet the demand of the device, the device becomes under powered and will not operate properly. The circuit diagram below shows the design of the power supply adopted for this work [9].

The power supply required for this circuit is regulated $5 \mathrm{v}$ dc. The component for the design include;

$$
\begin{aligned}
& \text { * The 9v dc battery } \\
& \text { * Voltage regulator } \\
& \text { * Capacitors }
\end{aligned}
$$

The 9v dc battery: The input voltage to the LM7805 should be at least $2 \mathrm{v}$ greater than the required $5 \mathrm{v}$ output according to its rating from the datasheet; hence it requires an input voltage of atleast $7 \mathrm{v}$. Hence, $9 \mathrm{v}$ battery was chosen with current rating of $1 \mathrm{~A}$ for convenience.

Voltage regulator: The LM7805 voltage regulator IC was used since we needed 5v. The IC is a three pin IC, having the input pin, ground pin and output pin. The regulator contains reference voltage element (zener diode), sampling element, error amplifier element and power control element. Its rating are; Input voltage, $7 \mathrm{v}-35 \mathrm{v}$, Current rating, $I_{c}=1 \mathrm{~A}$ and Output voltage range, $V_{\max }=$ $5.2 \mathrm{~V}, V_{\min }=4.2 \mathrm{~V}$

Capacitor: The design is intended to be portable which is why a 9v battery is chosen. The LM7805 chip is a 5v voltage regulator which is capable of taking input voltage of the range $7-70 \mathrm{v}$ (data sheet). Also, the $0.01 \mu \mathrm{F}$ capacitor is specified by datasheet of LM7805. This is to make less severe transients in output voltage due to changes in load.

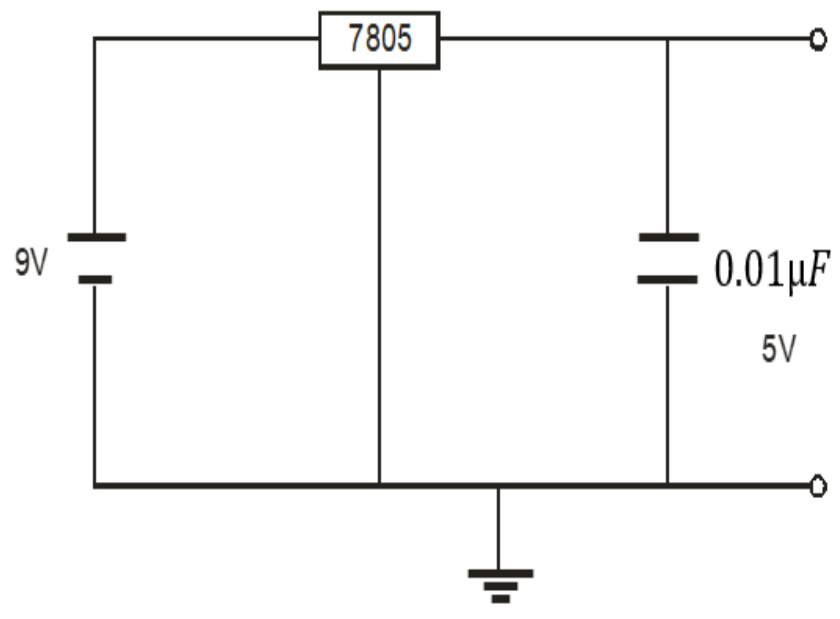

Figure 1: voltage regulator

\section{$2.2 \quad$ Input buttons}

Input buttons are used in this design to send specific commands to the micro-controller. When the controller receives these commands, it executes certain functions in this program. These functions include: 
Enroll - This subroutine is used to store the fingerprints of students in its database.

Match - This function is used to match fingerprints.

Delete - This is used to delete fingerprints from database.

Print - This subroutine is used to print out list of students that come to class together with date and time that each student came to class.

Reset - This button does not execute any function but restarts the controller.

Download- The button is used to extract the attendance information from the fingerprints module, into the MATLAB environment for analysis and storage.

Each of the buttons is connected to the controller as shown below.

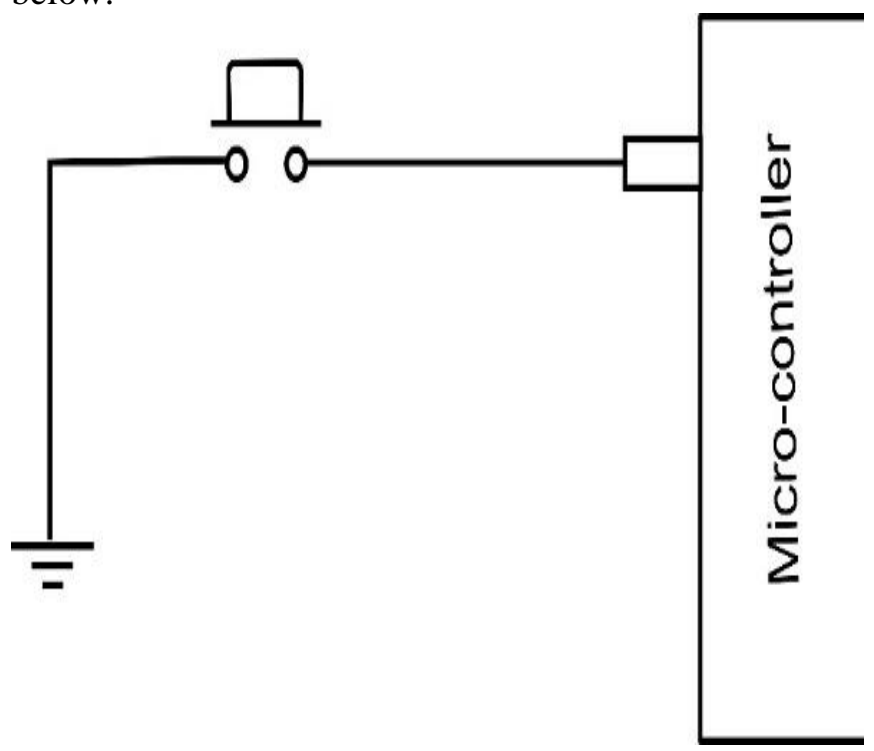

Figure 2: Input button

Buttons are usually connected to controller using either pull-up resistor or pull-down resistor, but in this case, neither pull-up resistor nor pull-down resistor was used. This is because, the controller used in this design process pull-up resistors on all buttons that can be configured as input or output (see data sheet). And so, to use these pull-up resistors, they have to be activated from software which is what was done in this project. The figure below shows how a button can be connected to a controller using pull-up or pull-down resistors.

\subsection{R305 Fingerprints Module}

The fingerprints module used in this design is a R305 fingerprints module. According to the manufacturers' manual, the module includes three major parts; fingerprint enrollment, fingerprints matching and fingerprints delete. When enrolling, the user enters his finger twice. The system will process the two times finger images, generates a template of the finger based on the processing results and store the template. When matching, the user enters the finger through the optical sensor and system will generate a template of the finger and compare it with the templates of the finger library. Also, when deleting, the module wipes out images of the fingerprints already stored in its memory.

The protocol for communication between the module and the controller is Enhanced Universal Synchronous Asynchronous Receiver Transmitter (EUSART). By pressing the appropriate button, certain functions are executed. These functions uses the EUSART communication protocol to send packets of data in 16bits (see data sheet), or appropriate command for enrolment, matching or deleting to the fingerprints module. The function codes used in this design can be seen at the appendices section.

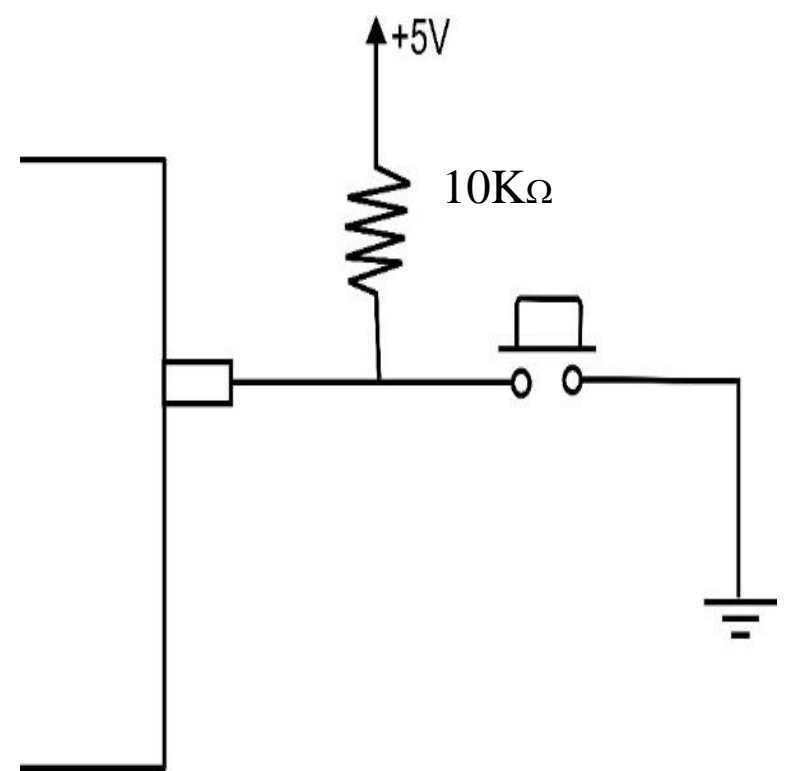

Figure 3: Pull-up resistor

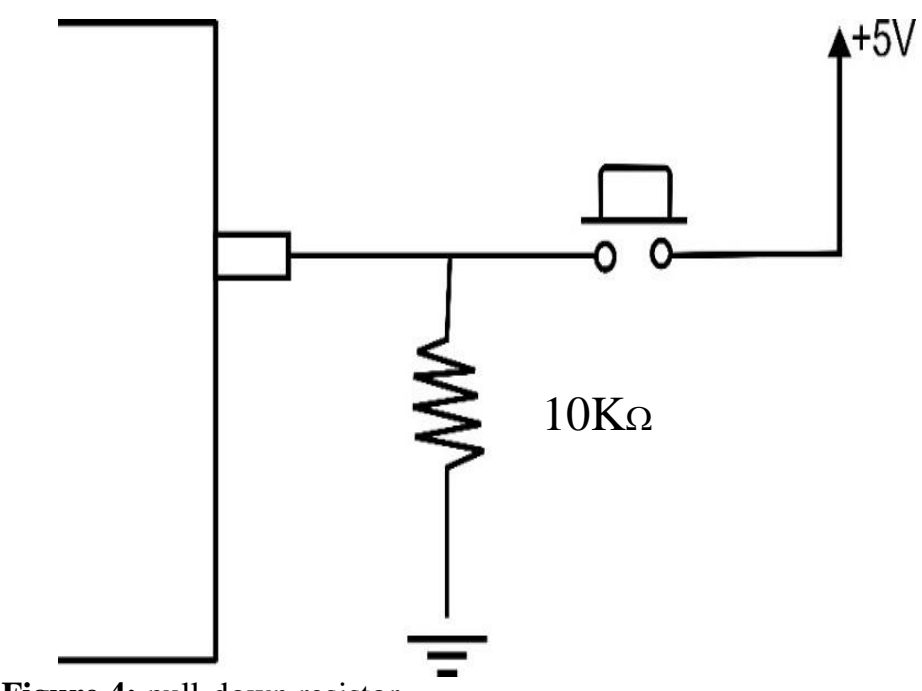

Figure 4: pull-down resistor 


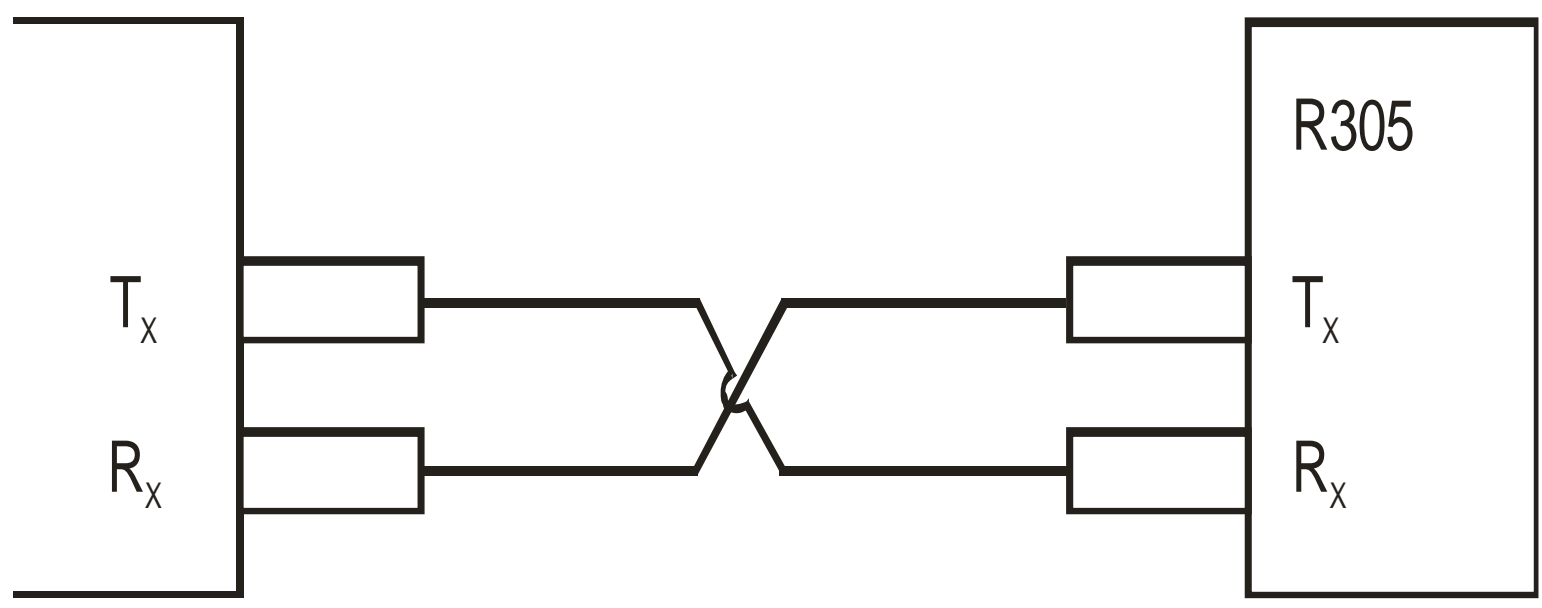

Figure 5: Connection diagram between R305 fingerprints module and the controller

\subsection{Real Time Clock (DS1307)(RTC)}

In this design, a DS1307 chip, together with a $3.1 \mathrm{v}$ battery cell is used to keep track of time. This is because, information such as date lecture held and exact time the student entered his fingerprint is collected and logged into a memory card or the memory of the R305 fingerprints module. The RTC is connected to the controller as shown below. The RTC keeps track of time even when the battery to the controller is removed.

This is because it has its own battery. RTC uses a protocol for communication referred to as inter-integrated communication protocol, often times shortened as $i 2 c$ or $i^{2} c$ communication protocol.
The connection diagram needed to achieve this communication protocol is shown in figure 3.7. In this mode of communication, several devices that support this communication protocol can be connected simultaneously as shown above. Communicating to any device is easy. The address of the device, among the so many connected, is first sent, then the device becomes active and communication with it is established.Most times more than a dozen devices can be connected using $i^{2} c$ communication protocol. The advantage of this communication protocol is that, no matter the number of devices connected to the communication bus, only two wires are required to communicate with all the devices.

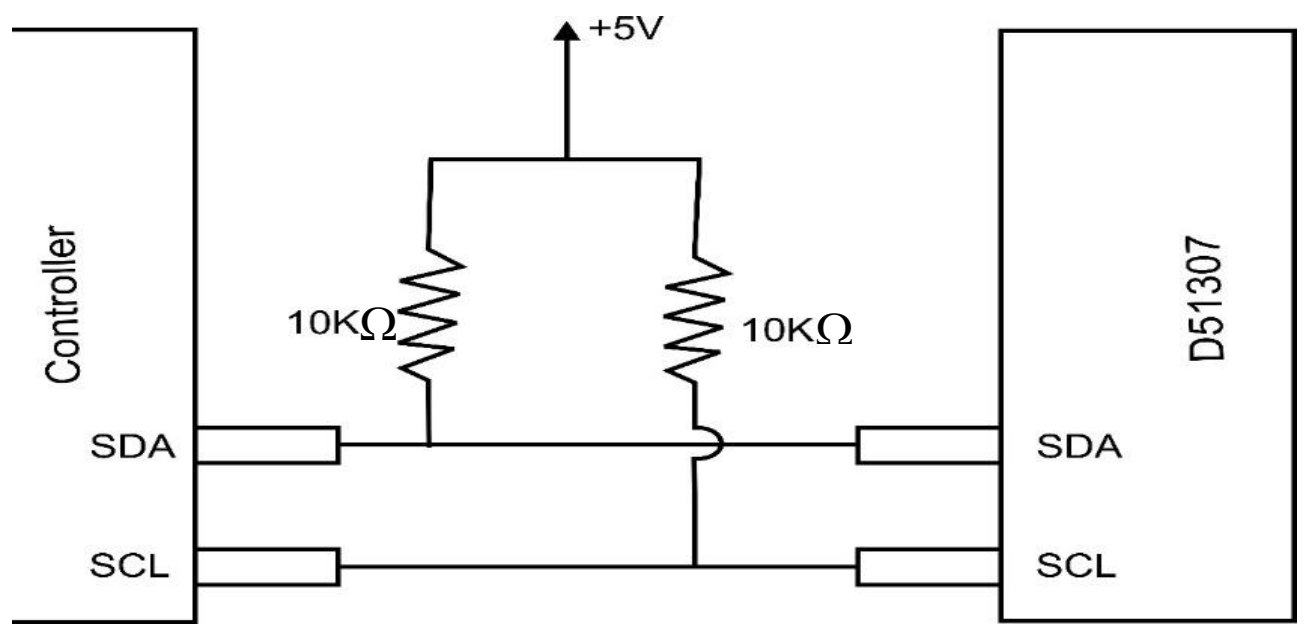

Figure 6: Interfacing DS1307 with controller

\section{$2.5 \quad 20 \times 4$ Liquid Crystal Display (LCD)}

The LCD module used in this design is QARASS 2004A, 20×4LCD module and has a SPLC78001 or equivalent controller which is a chip on board (COB) (TINSHARP LCD module data sheet, 2009).
The LED is used to display information of what the user is doing and what the system is doing, making this design user friendly. The circuit diagram below shows how the LCD is interfaced with the controller. 


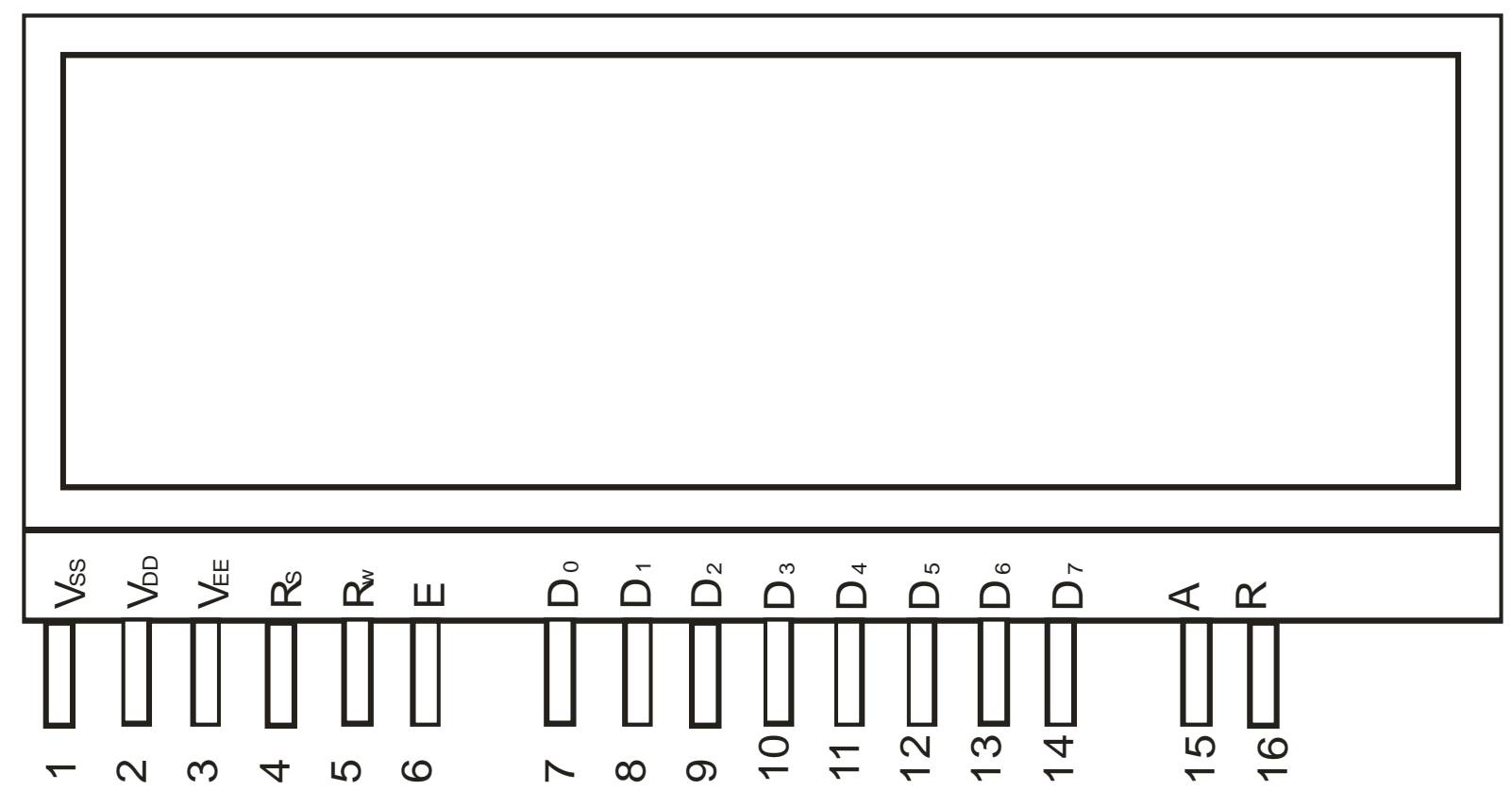

Figure 7: Pin out diagram for $20 \times 4$ LCD module

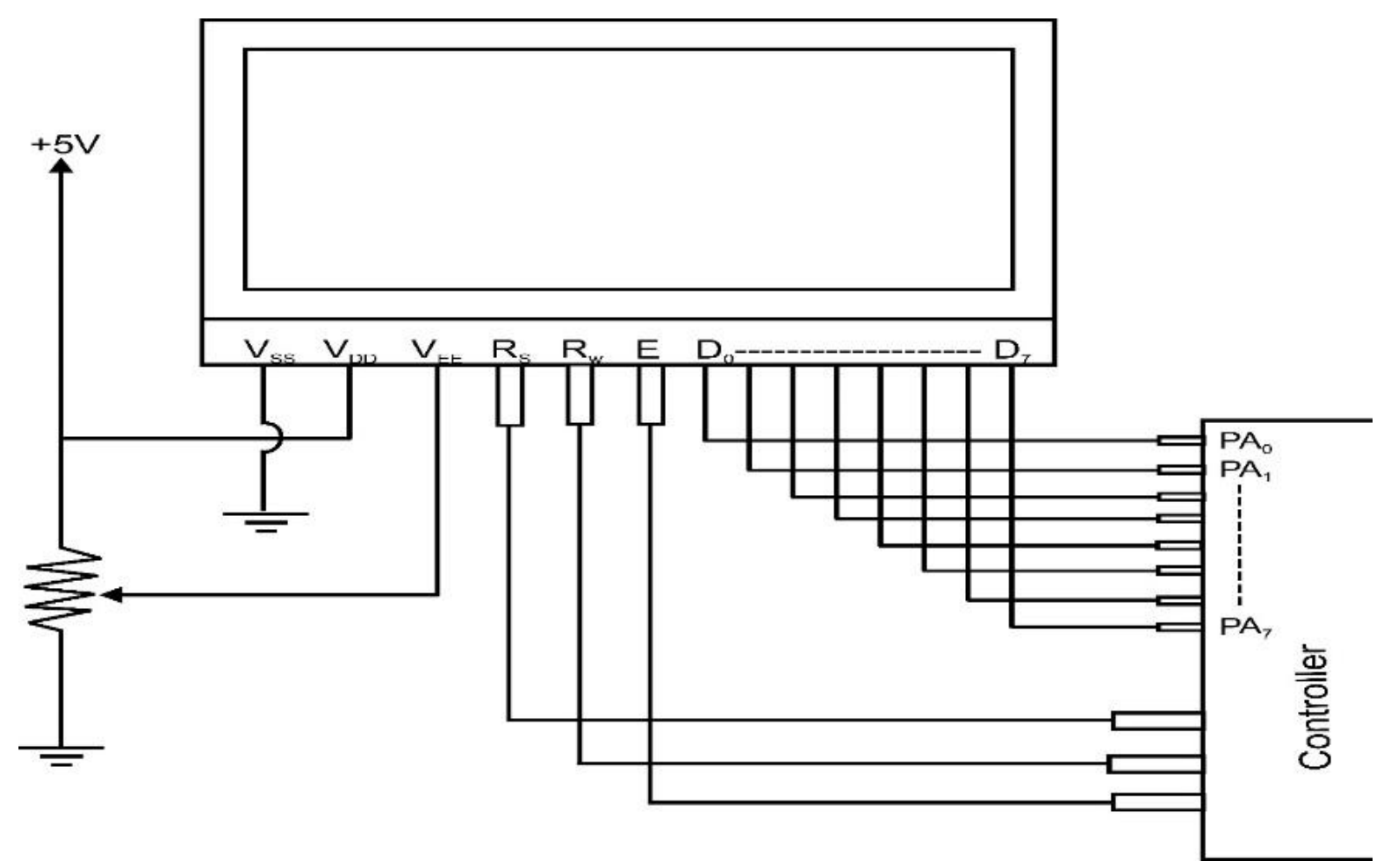

Figure 8: interfacing $20 \times 4$ LCD module with controller

The COB of the LCD module recognizes two types of commands. The first type of command configures the LCD module and is initiated by sending the following signals from the controller.

a) Hold the enable pin HIGH.

b) $R_{S}$ pin is given a digital LOW.

c) $R_{W}$ pin is given a digital HIGH.

Nigerian Journal of Technology (NIJOTECH) d) Send the appropriate configuration byte (8-bits) through pins $D_{0}-D_{7}$ to the COB of the LCD module (see data sheet).

e) Finally, clear the enable bit.

Send the appropriate configuration byte (8-bits) through pins $D_{0}-D_{7}$ to the COB of the LCD module (see data sheet). 
f) Finally, clear the enable bit.

The second type of command is used to display characters on the screen. In achieving this feat, the following process is adequate.

a) Hold the enable pin HIGH.

b) $R_{S}$ pin is given a digital HIGH.

c) $R_{W}$ pin is given a digital HIGH.

d) Send the appropriate configuration byte representing the character to be displayed through pins $D_{0}-D_{7}$ to the COB of the LCD module (see data sheet).

e) Finally, clear the enable bit.

\subsection{The Microcontroller}

The controller used in this design is Atmega644 from ATMEL. The controller has a Read Only Memory (ROM) of 64kbytes and Random Access Memory (RAM) of 8kbytes. This controller was chosen for this design because of its high ROM and RAM capacities. The huge ROM and RAM makes it possible to comfortably accommodate the codes which are really large [10].

\subsection{Circuit Diagram:} shown below.

The complete circuit diagram for this system is

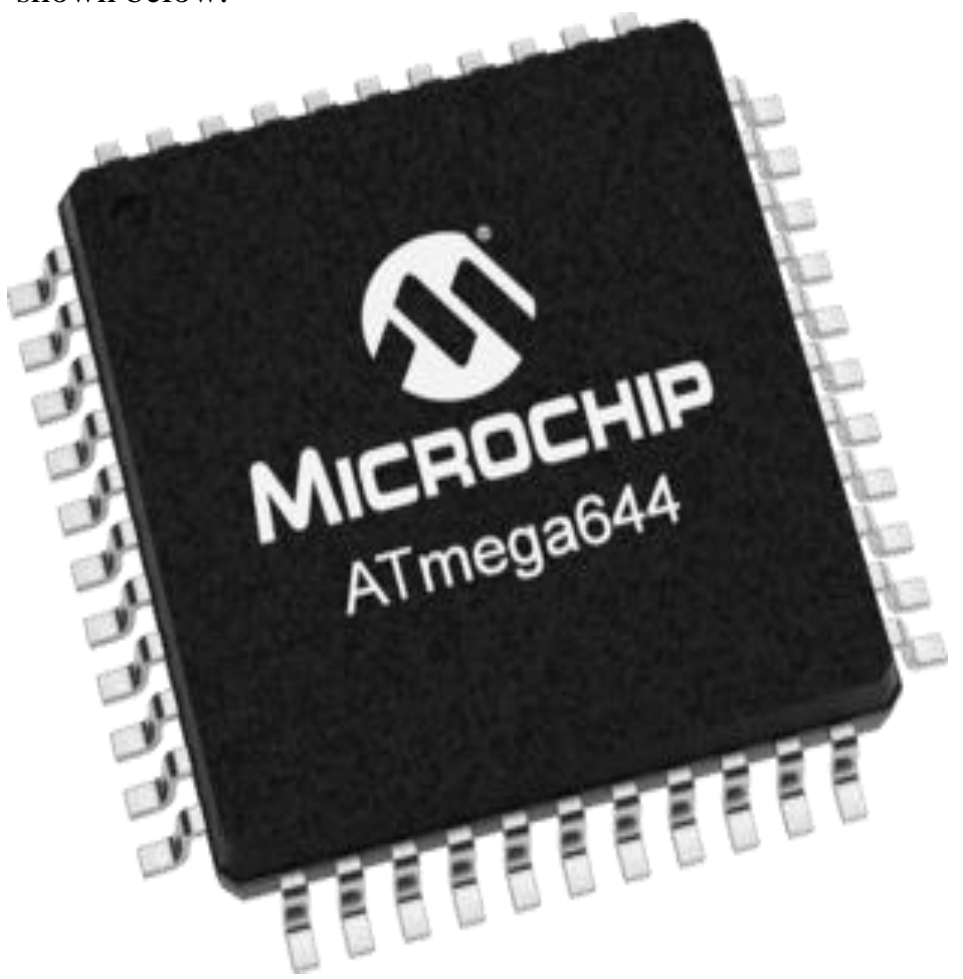

Figure 9: ATmega644

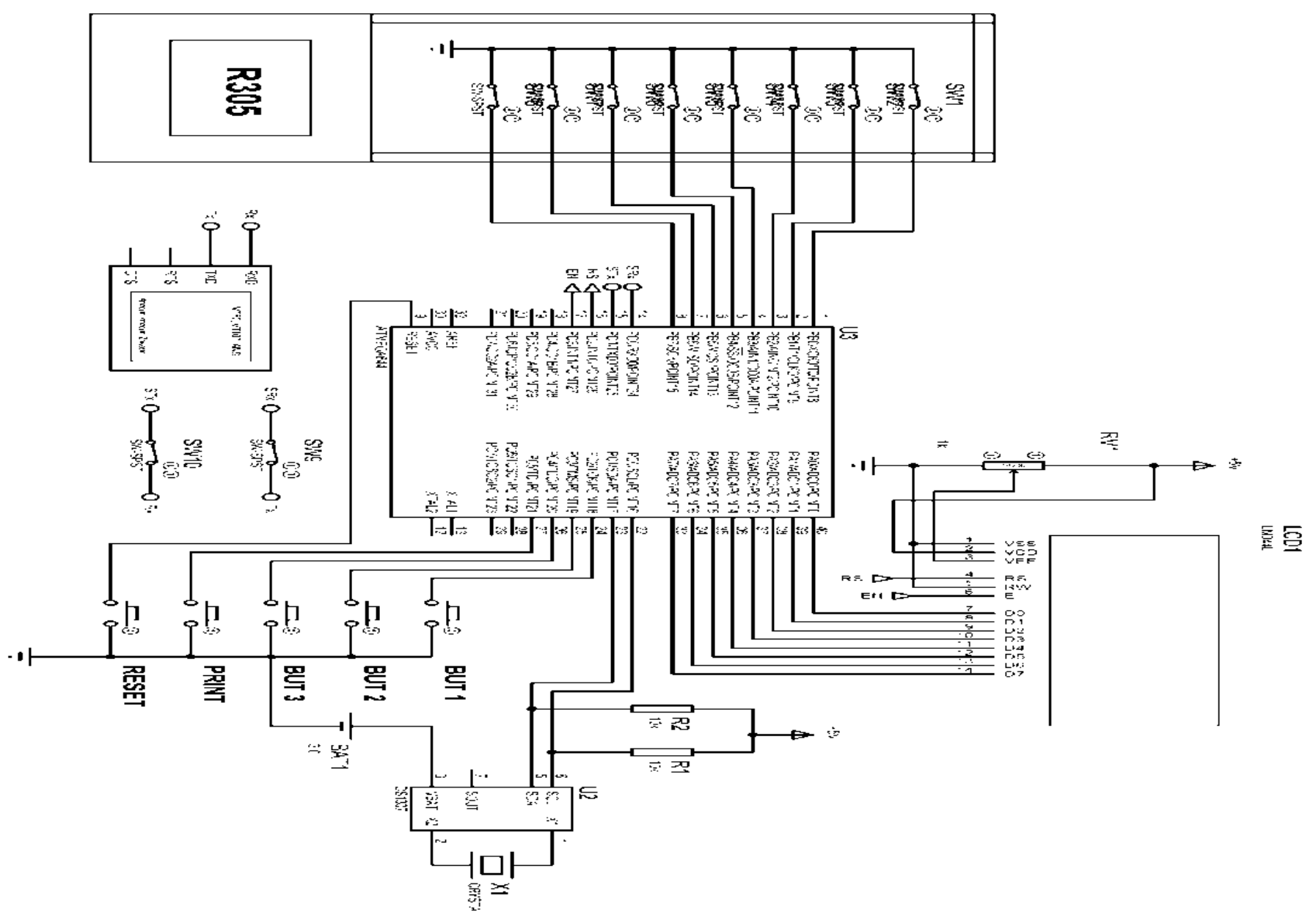

Figure 10: Circuit Diagram of Design 


\subsection{Principle of Operation}

In this design, the "Enroll" button is used to enroll students who are required to enter their fingerprints twice. If successful, the registration number of the student is matched with his fingerprints and stored. After registering all students, then the system is taken to the class or lecture hall ready to take attendance.

To take attendance of students present, the "Match" button is pressed. When this is done, the students will be required to place their fingers on the module until their registration number appears on the LCD screen. This means that student has been successfully matched, and the time he entered his finger is stored. The system is then passed on to the next student until all the students have entered their fngers. After the class, the lecturer takes the system to a computer, connects it to the computer running the fingerprints software. Using the download button, the lecturer will successfully extract the attendance record for the class. And at the press of the "print" button, the system prints out the date for that lecture and all the students present in that class with exact time they entered their finger prints. The system is programmed to store lectures held for more than fifty (50) different lectures, or until the computer runs out of memory

\subsection{SIMULATION RESULTS DISCUSSION}

The simulation of the prototype was done on a proteus professional software, using $\mathrm{C}++$ computer programming language, and attendance of students for four days were test-run and obtained as follows;

75\% AND ABOVE = ELIGIBILITY FOR WRITING EXAM TOTAL NUMBER OF REGISTERED STUDENTS: 24

STUDENTS ELIGIBLE FOR EXAM:

S/N REG NO. ATTENDANCE

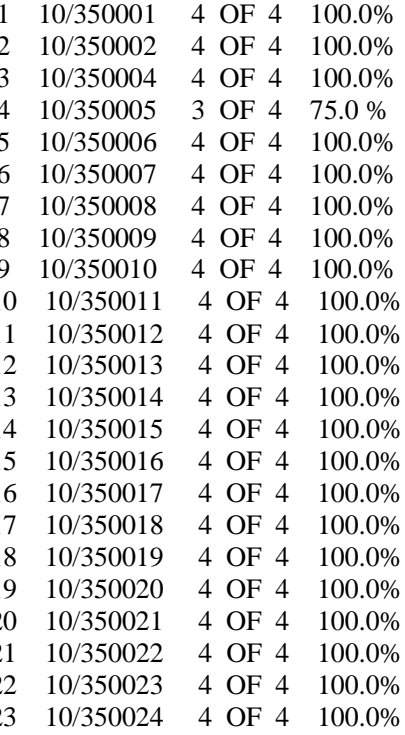

STUDENTS NOT ELIGIBLE FOR EXAM:

\begin{tabular}{cccc} 
S/N & REG NO. & \multicolumn{2}{c}{ ATTENDANCE } \\
-1 & $10 / 350003$ & 2 OF 4 & $50.0 \%$
\end{tabular}

After the construction was complete, the following steps were taken to test-run the module.

1. Eight students were registered with the module. This eight students represents eight out of the ten finger of the human limbs

2. Two lecture from the same day but different times were held

3. On the first session of lecture only five students were in attendance while on the second day of lecture only three students were in attendance

The figures that follows gives illustrations of results obtained.

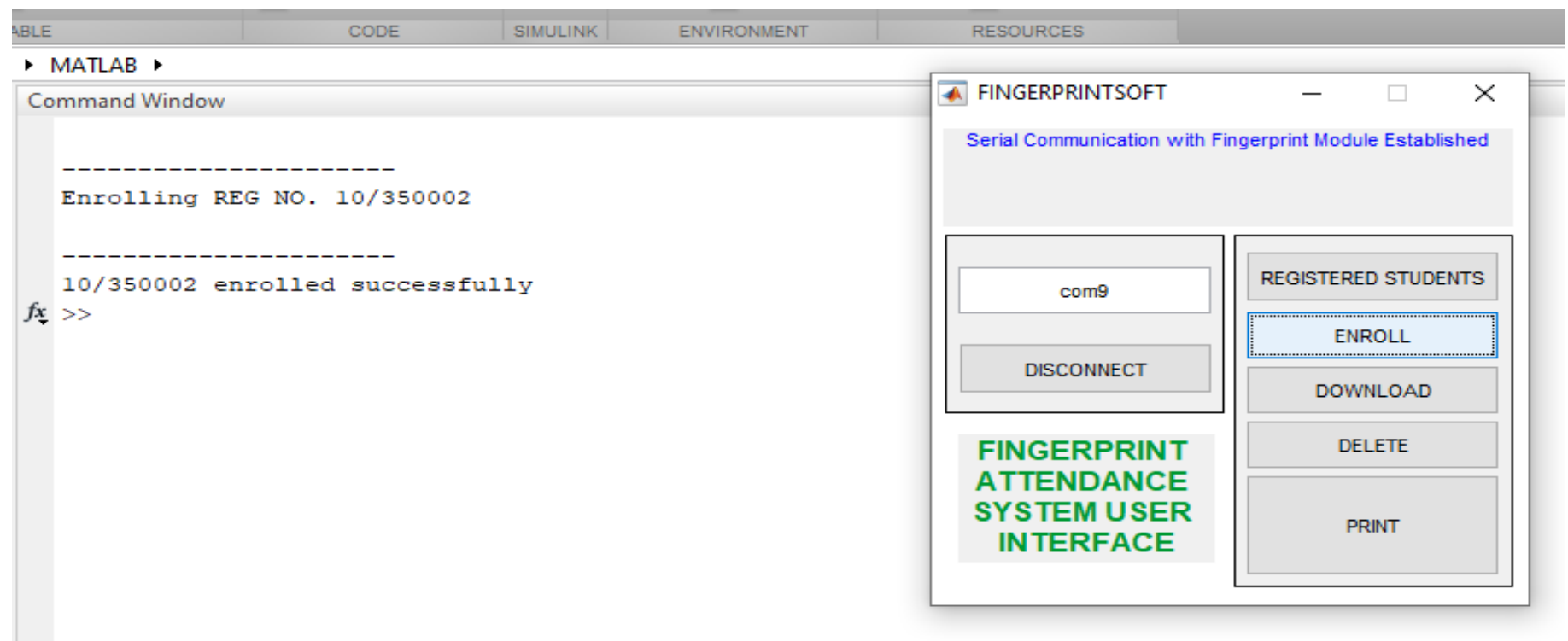

Figure 11: Successful enrolment 

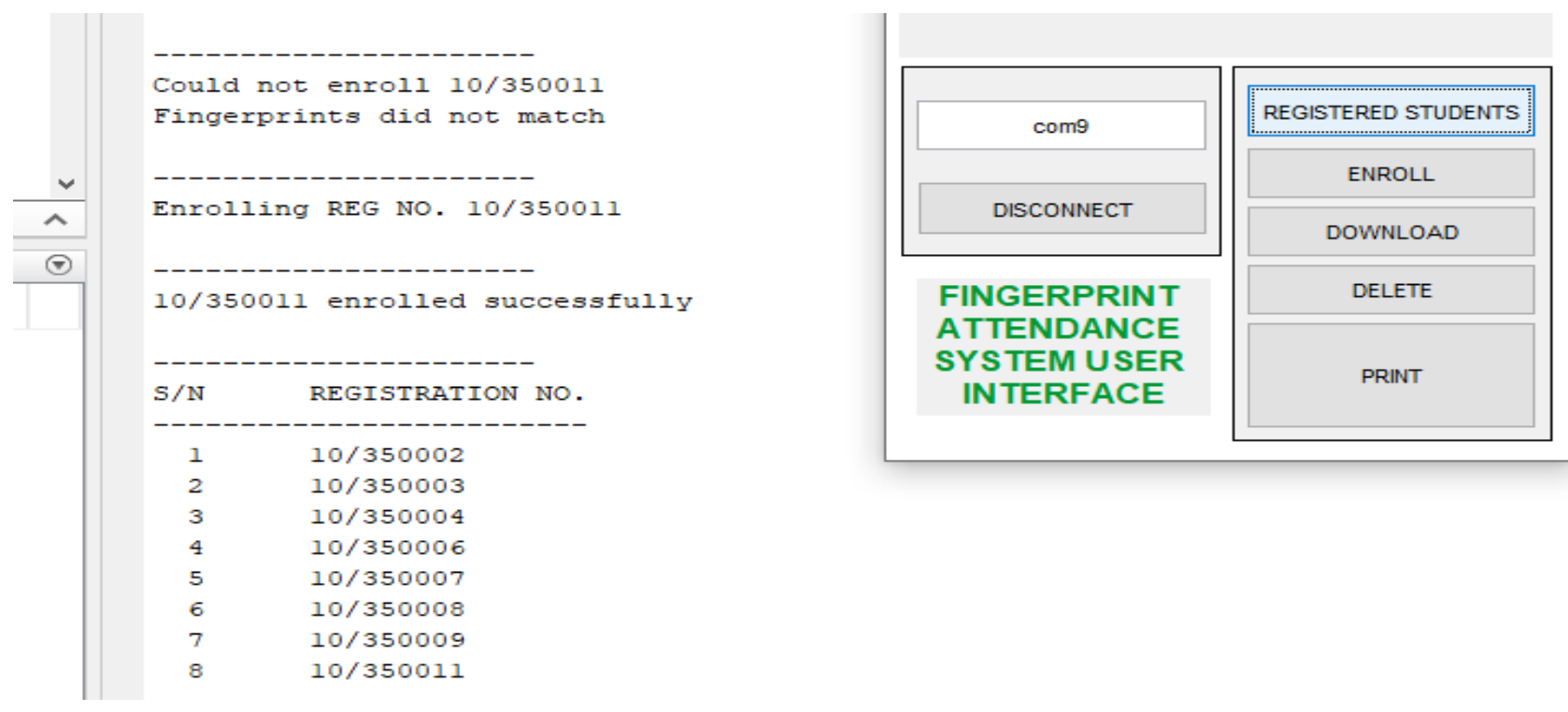

Figure 12: Enrolled eight students
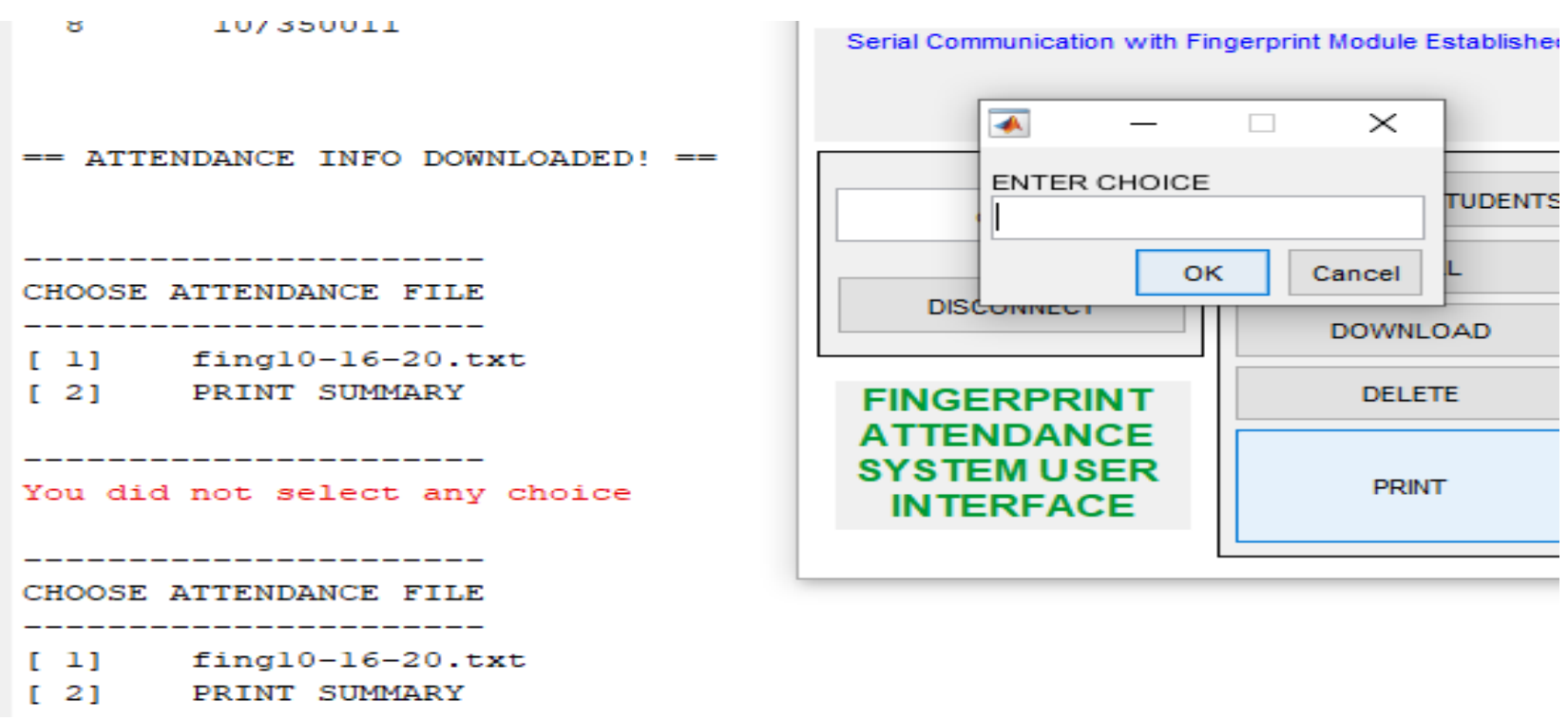

Figure 13: Check attendance

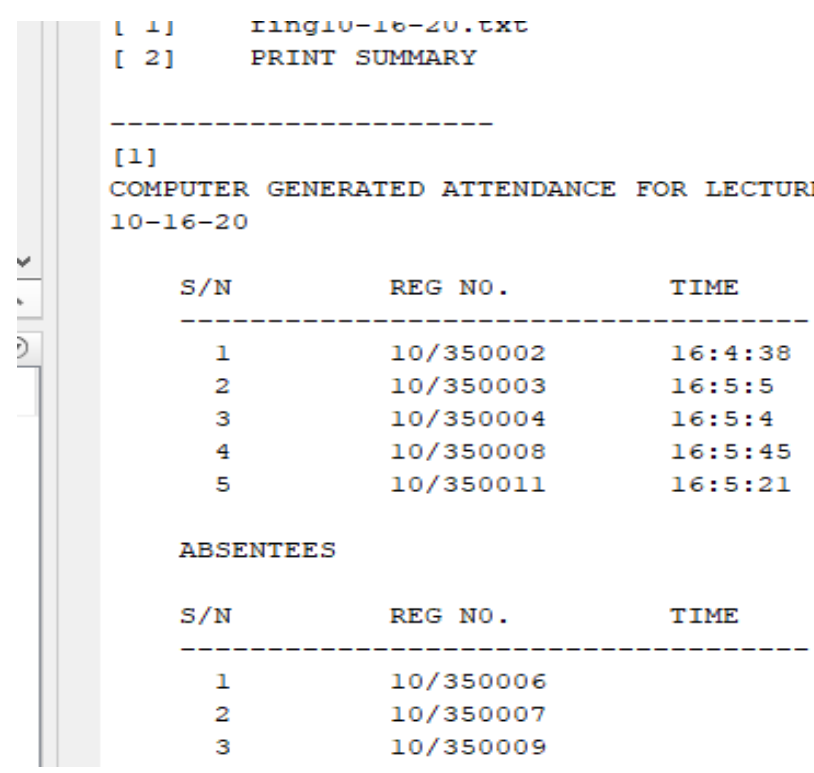

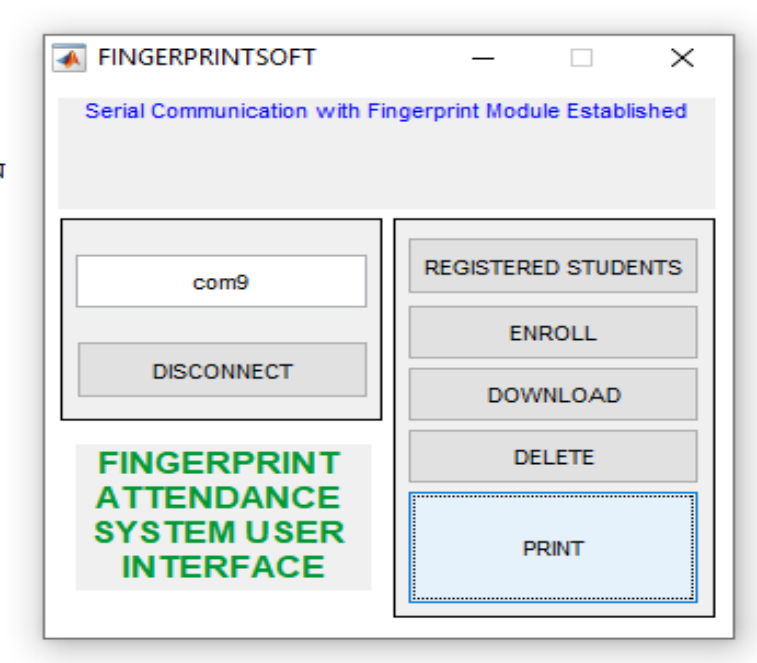


Figure 14: Attendance for first lecture displayed
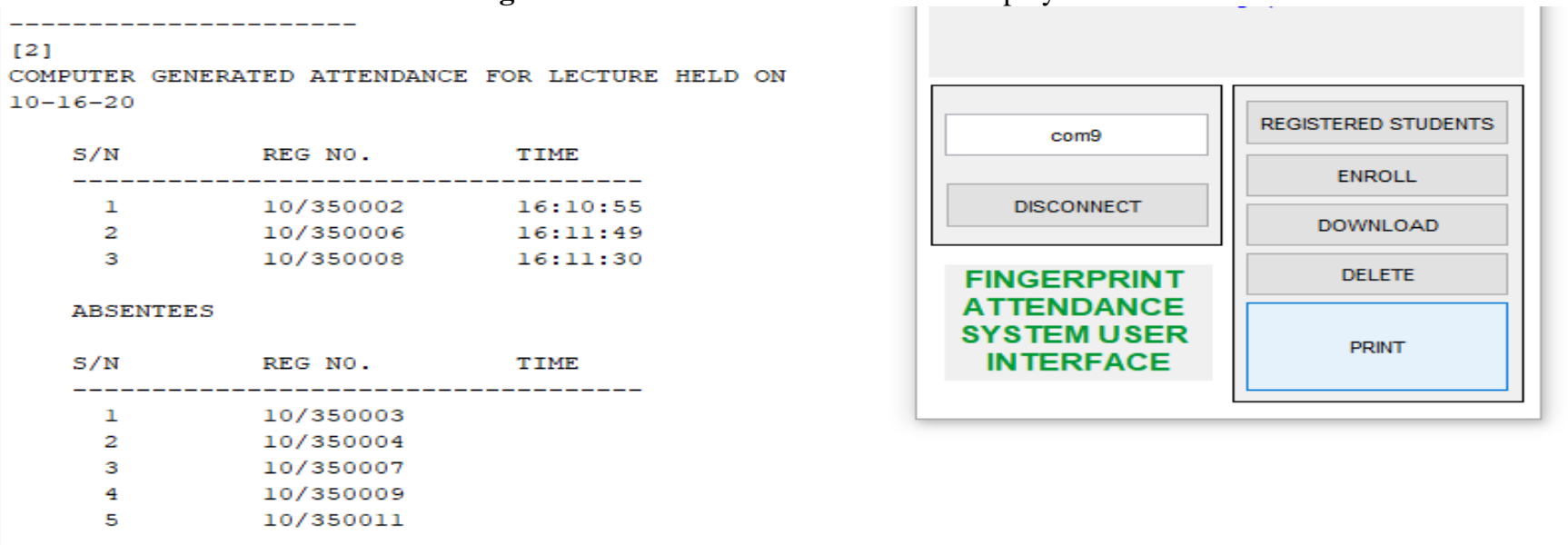

Figure 15: Attendance for second lecture displayed

\subsection{Discussions of Construction results}

Figure 11 shows the invoked GUI written using the MATLAB programming language. From the GUI, a font with the caption "Serial communication with the fingerprint module established" can be seen. This shows successful communication between the GUI and the fingerprint module.

When the fingerprint module is connected and the enroll button is clicked, the GUI prompts the user to enter the registration number of the student whose finger is to be captured while at the screen of the module displays the processes. Figure 12 is a display showing successful enrollment of the student

After enrolling eight students and confirming by clicking the registered students button, figure 13 suffices. The module is taken to class and registered students are expected to match their ID by placing their finger on the module. When this is done, attendance is taken with date and time automatically. If a finger that is not registered in the database is placed, nothing happens. If a registered student did not come to class, his attendance is not taken. After taking the attendance, the attendance data was move (or download) from the memory of the module to the database in MATLAB using the GUI. This is done by clicking the download button in the GUI.

To display the attendance of a particular lecture, the print button of the GUI was clicked. The appropriate option was selected by entering the correct number into the GUI that pops up (See figures 13). If multiple attendance have been taken, then the number corresponding to summary is clicked as shown in figure 14 and figure 15 . The attendance analyses results are obtained as shown below

CHOOSE ATTENDANCE FILE

[1]

16-20.txt

[2]

16-20_001.txt

[3]

SUMMARY
[1] COMPUTER GENERATED ATTENDANCE FOR LECTURE HELD ON $10-16-20$

$\begin{array}{ccc}\text { S/N } & \text { REG N0. } & \text { TIME } \\ -1 & 10 / 350002 & 16: 4: 38 \\ 2 & 10 / 350003 & 16: 5: 5 \\ 3 & 10 / 350004 & 16: 5: 4 \\ 4 & 10 / 350008 & 16: 5: 45 \\ 5 & 10 / 350011 & 16: 5: 21\end{array}$

ABSENTEES

\begin{tabular}{ccc} 
S/N & REG NO. & TIME \\
\hdashline 1 & $10 / 350006$ & \\
2 & $10 / 350007$ \\
3 & $10 / 350009$
\end{tabular}

[2] COMPUTER GENERATED ATTENDANCE FOR LECTURE HELD ON $10-16-20$

\begin{tabular}{ccc} 
S/N & REG NO. & TIME \\
\hdashline 1 & $10 / 350002$ & $16: 10: 55$ \\
2 & $10 / 350006$ & $16: 11: 49$ \\
3 & $10 / 350008$ & $16: 11: 30$
\end{tabular}

\section{ABSENTEES}

$\begin{array}{crr}\text { S/N } & \text { REG N0. TIME } \\ -1 & 10 / 350003 & \\ 2 & 10 / 350004 & \\ 3 & 10 / 350007 \\ 4 & 10 / 350009 \\ 5 & 10 / 350011\end{array}$

TOTAL ATTENDANCE FOR EACH STUDENT

\begin{tabular}{cccc} 
S/N & REG N0. & ATTENDANCE \\
\hdashline 1 & $10 / 350002$ & 2 of 2 & $100.0 \%$ \\
2 & $10 / 350003$ & 1 of 2 & $50.0 \%$ \\
3 & $10 / 350004$ & 1 of 2 & $50.0 \%$ \\
4 & $10 / 350006$ & 1 of 2 & $50.0 \%$ \\
5 & $10 / 350007$ & 0 of 2 & $0.0 \%$ \\
6 & $10 / 350008$ & 2 of 2 & $100.0 \%$ \\
7 & $10 / 350009$ & 0 of 2 & $0.0 \%$ \\
8 & $10 / 350011$ & 1 of 2 & $50.0 \%$
\end{tabular}


$75 \%$ = ELIGIBILITY FOR WRITING EXAM

TOTAL NUMBER OF REGISTERED STUDENTS: 8

STUDENTS ELIGIBLE FOR EXAM:

\begin{tabular}{cccc} 
S/N & REG N0. & ATTENDANCE \\
\hdashline 1 & $10 / 350002$ & 2 of 2 & $100.0 \%$ \\
2 & $10 / 350008$ & 2 of 2 & $100.0 \%$
\end{tabular}

STUDENTS NOT ELIGIBLE FOR EXAM:

$\begin{array}{cccc}\text { S/N } & \text { REG N0. } & \text { ATTENDANCE } \\ -1 & 10 / 350003 & 1 \text { of } 2 & 50.0 \% \\ 2 & 10 / 350004 & 1 \text { of } 2 & 50.0 \% \\ 3 & 10 / 350006 & 1 \text { of } 2 & 50.0 \% \\ 4 & 10 / 350007 & \text { 0 of } 2 & 0.0 \% \\ 5 & 10 / 350009 & 0 \text { of } 2 & 0.0 \% \\ 6 & 10 / 350011 & 1 \text { of } 2 & 50.0 \%\end{array}$

\subsection{CONCLUSION}

The Fingerprints Attendance System with Datalogin was designed, simulated and implemented/constructed, and was able to address the attendance issues in Adamawa State University, Mubi by the following means: no time waste as the attendance is taken during lecture without intervention of the lecturer, managing the attendance is automated, no chance for buddy signing, real time attendance capture, evaluate level of attendance for students automatically. Also, this system can easily be apply for examination attendance and monitoring. The main feature of the system is the low cost.

\section{REFERENCES}

[1] Shila, S. "A DoG based approach for fingerprint Image enhancement". Ph.D Thesis, Department of Computer Science and Engineering, NIT Rourkela. 2011.

[2] Caldwell, T. "Market report: "border biometrics". Biometric Technology, 5,(2015), 5-11.

[3] Jyoti, R., Gupta, P.C. and Sharmarvind, C. "Fingerprint-based Identification System-Asurvey". International journal of Computer Technology and Electronic Engineering, 1(3), (2016).

[4] Chaudhari, A.S., Patnaik, G.K. and Patil, S.S. "Implementation of Minutiae Based Fingerprint Identification System Using Crossing Number Concept". Informatica Economica, 18(1), (2014), 17-26.

[5] Iloanusi, O. N., "Fingerprint Marching using Minutiae Quadruplets". Nigerian Journal of Technology, 29(1), (2010), 86-93.

[6] Hussain, A., Hammad, M., Hafeez, K. and Zainab, T. "Programming a Microcontroller". International Journal of Computer Applications, 155(5), (2016), 21-26.

[7] Padgavhankar V.A. and Mohod W.S. 'Experimental Learning of Digital Power Controller for
Photovoltaic Module Using Proteus VSM". Journal of Solar Energy, Journal of Solar Energy, (2014),18.

[8] Bansal, R., Sehgal, P. and Bedi, P. "Minutiae extraction from fingerprint images-a review". International Journal of Computer Science 8(5), (2011), 74-85.

[9] Nur Izzati Zainal, Khairul Azami Sidek, Teddy Surya Gunawan, Hasmah Mansor, and Mira Kartiwi, "Design and Development of Portable Classroom Attendance System Based on Arduino and Fingerprint Biometric". Information and Communication Technology for the Muslim World (ICT4M), $5^{\text {th }}$ International Conference, 2014.

[10] Axelson, J., 1997. The Microcontroller Idea Book: Circuits, Programs \& Applications Featuring the 8052-BASIC Microcontroller. lakeview research llc. 